\title{
Los conductores adolescentes tienen más riesgo de morir en accidentes de tránsito cuando transportan pasajeros
}

\author{
Carrying passengers as a risk factor for crashes fatal to 16 and 17 years old drivers.
} Chen L, Baker SP, Braver ER, et al. JAMA 2000;283:1578-1582

\section{Objetivo}

Evaluar si el riesgo de muerte de conductores de 16-17 años está asociado al número y características de los pasajeros.

\section{Diseño}

Estudio sobre datos de todo el territorio de los EE.UU. provenientes de tres fuentes de registros de accidentes distintas y complementarias pertenecientes al gobierno federal, entre 1992 y 1997. Las bases de datos aportaron todos los accidentes fatales de EE.UU., el número de viajes estimado para conductores de 16 y 17 años según encuesta telefónica, y datos de una muestra representativa de toda la población del país sobre accidentes con conductores de 16-17 años.

\section{Participantes}

El foco del estudio fueron todos los conductores de 16-17 años que protagonizaron un accidente. Algunos análisis incluyeron un grupo control con conductores de 30 a 59 años. Los accidentes fatales primariamente incluidos fueron aquellos que involucraron la muerte del conductor hasta 30 días de ocurrido el hecho. A partir de estos se estudiaron las características de los ocupantes de los vehículos.

\section{Medición de resultados principales}

Muertes cada 10 millones de viajes: probabilidad de muerte de un conductor de 16-17 años, teniendo en cuenta la edad, sexo, hora y número de ocupantes. Muertes cada 1000 accidentes de conductores 16-17 años según edad y sexo de los ocupantes. Esta medida representa el resultado de muchas variables que podrían estar afectando la conducta de los conductores asociada a la presencia de pasajeros. Se definió riesgo relativo (RR) como la mortalidad del grupo de 16-17 años en relación a la del grupo control.

\section{Resultados}

Tasa de muertes/10 millones de viajes: los conductores adolescentes tienen más riesgo de morir que los adultos y su riesgo aumenta proporcionalmente al número de pasajeros, 5.61 con tres, contra 0.47 de los conductores adultos. Los hombres tienen más riesgo que las mujeres. El mayor riesgo (21.88) ocurre de 0 a 6 AM y con pasajeros.

Tasa de muerte/1000 accidentes: el riesgo de muerte de los conductores adolescentes es mayor con pasajeros adolescentes con respecto a conducir solos o con pasajeros mayores 2.9 (IC95\% 2.46 a 3.90) contra 1.44 conduciendo solos. También aumenta el riesgo llevar pasajeros varones: 2.63. El efecto de pasajeros varones es similar para conductores de ambos sexos y aumenta con el número de los mismos.

RR: Entre los conductores adolescentes de 16 años el RR de morir comparado con viajar solo es: con un pasajero 1.39 (IC95\% 1.24 a 1.55), con dos 1.86 (IC95\% 1.56 a 2.20), con tres o más 2.82 (IC95\% 2.27 a 3.50 ) (los resultados similares en los conductores de 17 años).

\section{Conclusión}

Los conductores de 16-17 años sufren una tasa de muertes mayor que la de los adultos. Este riesgo es mayor cuanto más sean los pasajeros; y también aumenta con pasajeros hombres y adolescentes.

\section{COMENTARIO}

Los accidentes de tránsito son la principal causa de muerte entre los adolescentes. La incidencia de muerte de conductores de 16-17 años es mayor que para adultos independientemente del sexo. Como en otros estudios los conductores varones y las horas de la madrugada aumentan el riesgo de muerte ${ }^{1}$. ¿Pero, por qué transportar pasajeros aumenta el riesgo de muerte entre los conductores adolescentes? Las conductas de riesgo (alta velocidad, menor distancia de frenado, maniobras bruscas, traspasar carriles, ir siguiendo la luz amarilla, etc.) están fuertemente asociadas con la presencia de pares. ${ }^{2,3} \mathrm{El}$ efecto de llevar pasajeros no varía con la hora. Al contrario, los conductores adultos tienen menor riesgo de muerte cuando transportan gente. Aunque no reportado en el artículo, el consumo de drogas y alcohol es un factor que también está asociado a la presencia de pasajeros y a la muerte de los conductores. Se ha comprobado que los adolescentes son más susceptibles que los adultos a errores en el manejo y conductas arriesgadas con igual concentración de alcohol en sangre. ${ }^{4}$ Aún así la contribución del alcohol a la muerte de adolescentes conductores es menos importante que para adultos en comparación a la inexpe- riencia, impulsividad, inmadurez y distracción con los pasajeros. ${ }^{5,6}$ La editorial referida a este artículo recomienda adoptar el sistema de "licencia de conducir graduada" que impone restricciones a los principiantes en las condiciones de manejo, y son quitadas progresivamente. ${ }^{7}$ Los estados o países que lo adoptaron lograron una disminución de accidentes de entre el 7 y $32 \%$. Básicamente crean distintos grados de licencia con restricciones como estar acompañados por adultos con licencia, o por los padres, viajar sin pasajeros, o tener prohibido los horarios nocturnos. ${ }^{8}$

Los accidentes y las muertes por accidentes de tránsito tienden a disminuir en el mundo desarrollado por el avance en la educación y sobre todo por los adelantos en tecnología automotriz y vial. Sin embargo, esto no es así para los conductores adolescentes. Ellos representan un riesgo para si mismos y para la sociedad porque operar un vehículo de motor es una actividad cognocitivamente muy compleja. Tal vez en nuestros países, donde implementar y controlar licencias graduadas sería imposible, deberíamos revisar la edad para otorgar el registro. A los médicos de atención primaria nos toca educar a los adolescentes y advertir a los padres sobre los factores de riesgo controlables.

Dr. Virgilio Petrungaro

Programa de Efectividad Clínica. Especialista en Clínica Médica. Sanatorio de La Providencia.

\section{Referencias}

1. Yang Mao, Jun Zhang, Glenn Robbins, et al. Factors affecting the severity of motor vehicle traffic crashes involving young drivers in Ontario. Injury Prevention 1997; 3:183-189. 2. Farrow JA. Young driver risk taking: a description of dangerous driving situations among 16-19 years old drivers. Int. j. Addict. 1987;22:1255-1267.

3. Mc. Kenna FP, Waylen AE, Burkes ME. Male and Female Drivers, How different are they? Reading, England: University of Reading, AA Foundation for Road Safety Research; 1998. 4. Mayhew DR, Warren RA, Simpson HM. Young Driver Accidents, Ottawa, Ontario: Traffic Injury Research Foundation; 1981

5. National Highway Traffic Safety Administration. Fatality Analysis Reporting System, 1998. http://www-fars.nhtsa.dot.gov/www/query.htlm. Accessed January 15, 2000.

5. Williams AF, Preusser DF, Ulmer RG, Weinstein HB. Characteristics of fatal crashes of 16 years old drivers: implications for licensure po licies. J Public Health Policy. 1995; 16: $347-360$.

7. Foss DF. Reducing fatal crash risk among teenaged drivers. Structuring an effective graduated licensing system. JAMA 2000;283:1617-8.

8. Phebo L, Dellinger AM. Young driver involvement in fatal motor vehicle crashes and trends in risk behaviors, United States, 1988-95. Injury Prevention 1998; 4:284-287. 